\title{
O papel do Sistema Único de Saúde no combate à sindemia global e no desenvolvimento de sistemas alimentares sustentáveis
}

\author{
The role of the Brazilian Unified Health System in combating the \\ global syndemic and in the development of sustainable food systems
}

\author{
Alisson Diego Machado (https://orcid.org/0000-0002-1564-5405) ${ }^{1}$ \\ Ana Maria Bertolini (https://orcid.org/0000-0002-6833-8249)2 \\ Letícia da Silva Brito (https://orcid.org/0000-0001-6982-505X) ${ }^{3}$ \\ Mirelly dos Santos Amorim (https://orcid.org/0000-0003-1031-7290) ${ }^{4}$ \\ Mônica Rocha Gonçalves (https://orcid.org/0000-0002-9228-6848) ${ }^{5}$ \\ Raquel de Andrade Cardoso Santiago (https://orcid.org/0000-0002-6894-2008) ${ }^{6}$ \\ Dirce Maria Marchioni (https://orcid.org/0000-0002-6810-5779) ${ }^{7}$ \\ Aline Martins de Carvalho (https://orcid.org/0000-0002-4900-5609) ${ }^{7}$
}

${ }^{1}$ Departamento de Clínica Médica, Disciplina de Nefrologia, Faculdade de Medicina, Universidade de São Paulo. Av. Dr. Enéas de Carvalho Aguiar 255 sala 7115, Cerqueira César. 05403-000 São Paulo SP Brasil. alissondmachado@ gmail.com

${ }^{2}$ Departamento de Saúde Ambiental, Faculdade de Saúde Pública, Universidade de São Paulo. São Paulo SP

Brasil.

${ }^{3}$ Departamento de Biologia, Universidade de Aveiro. Aveiro Portugal.

${ }^{4}$ Escola Paulista de

Medicina, Universidade Federal de São Paulo. São

Paulo SP Brasil.

${ }^{5}$ Núcleo de Apoio às

Atividades de Cultura e Extensão Sustentarea, Faculdade de Saúde Pública, Universidade de São Paulo.

São Paulo SP Brasil.

${ }^{6}$ Faculdade de Nutrição,

Universidade Federal de

Goiás. Goiânia GO Brasil.

${ }^{7}$ Departamento de

Nutrição, Faculdade de

Saúde Pública, Universidade

de São Paulo. São Paulo SP

Brasil.

\begin{abstract}
The undernutrition and obesity pandemics associated with climate change are a global syndemic. They have a point of convergence, which is the unsustainable current food systems. This paper aims to discuss the role of public health policies, particularly the Brazilian Unified Health System (SUS) in the context of Primary Health Care, in combating the global syndemic and in the development of sustainable food systems. In this scenario, the National Food and Nutrition Policy is a leading intersectoral tool for an adequate and healthy diet and food and nutrition security. Also, the Dietary Guidelines for the Brazilian population is a strategic tool to support food and nutrition education. We highlight the need to articulate health, agriculture, and environmental policies to achieve sustainable development. Thus, SUS can be the arena to promote the main discussions on this topic, potentiating individual, group, and institutional actions to provide a fairer, healthy, and sustainable food system.
\end{abstract}

Key words Unified Health System, Health policy, Food and nutrition security, Sustainable development
Resumo As pandemias de desnutrição e obesidade, em conjunto com as mudanças climáticas, constituem uma sindemia global e apresentam um importante ponto de convergência, que é a insustentabilidade dos sistemas alimentares atuais. O objetivo deste artigo é discutir o papel de politicas públicas de saúde, particularmente do Sistema Único de Saúde (SUS) no âmbito da Atenção Primária à Saúde, no combate à sindemia global e no desenvolvimento de sistemas alimentares sustentáveis. Nesse contexto, a Politica Nacional de Alimentação e Nutrição se destaca como uma importante ferramenta intersetorial para a alimentação adequada e saudável e à segurança alimentar e nutricional. Além disso, o Guia Alimentar se apresenta como um instrumento estratégico de apoio às ações de educação alimentar e nutricional. De modo essencial, destaca-se a necessidade de articulação das políticas de saúde, agricultura e meio ambiente para que o desenvolvimento sustentável possa ser efetivado. Assim, o SUS tem capacidade de ser palco das principais discussões sobre essa temática, atuando como um potencializador de ações individuais, coletivas e institucionais para promover um sistema alimentar mais justo, saudável e sustentável.

Palavras-chave Sistema Único de Saúde, Política de saúde, Segurança alimentar e nutricional, Desenvolvimento sustentável 


\section{Introdução}

Atualmente, há três graves ameaças globais à saúde e à sobrevivência humana: as pandemias de desnutrição e de obesidade - que inclui sua relação com o desenvolvimento de doenças crônicas não transmissíveis (DCNT) - e as mudanças climáticas, que juntas constituem uma sindemia global $^{1}$. Antes vistas como condições divergentes e isoladas entre si, elas têm sido reconhecidas como fatores sinérgicos que coexistem no tempo e no espaço e compartilham determinantes sociais comuns em escala global. Além disso, partilham interações nos campos biológico, psicológico e social ${ }^{1}$.

Em sinergia com o que propõe o termo sindemia global, a Organização das Nações Unidas para a Alimentação e a Agricultura (FAO) reconhece o desafio latente da prevalência da má nutrição em todas as suas formas, incluindo a desnutrição, carências nutricionais específicas, sobrepeso, obesidade e DCNT associadas à alimentação ${ }^{2}$. No Brasil, apesar de inúmeros avanços realizados nesse campo, com especial atenção ao impacto do Programa Bolsa Família (PBF) na melhora da disponibilidade e aquisição de alimentos entre famílias de baixa renda ${ }^{3-5}$, ainda é possível encontrar lares brasileiros vivendo com algum grau de insegurança alimentar. Segundo a Pesquisa de Orçamentos Familiares (POF) 2017-2018, a insegurança alimentar grave esteve presente no lar de 10,3 milhões de pessoas (4,9\% da população), ou seja, faltavam alimentos entre todos os moradores, incluindo as crianças ${ }^{6}$.

Por outro lado, dados recentes da Pesquisa Nacional de Saúde (PNS) mostram que, em 2019, $25,9 \%$ da população maior de 18 anos estavam obesas (41,2 milhões de pessoas) e cerca de $60,3 \%$ da população nesse grupo etário (96 milhões de pessoas) estavam com sobrepeso ${ }^{7}$. Entre a POF 2002-2003 e a PNS 2019, a proporção de obesos na população com 20 anos ou mais de idade mais que dobrou, indo de $12,2 \%$ para $26,8 \% \%^{7}$. Paralelamente, os dados do Sistema de Vigilância de Fatores de Risco e Proteção para Doenças Crônicas por Inquérito Telefônico (Vigitel) de 2019 indicam um avanço das DCNT entre os brasileiros - 7,4\% possuem diabetes mellitus e $24,5 \%$ têm hipertensão arterial ${ }^{8}$.

É de amplo conhecimento que as DCNT são a principal causa de morbimortalidade no mundo, sendo a alimentação não saudável um de seus principais fatores de risco9. Tendo em vista seus efeitos negativos diretos na saúde, somados aos efeitos indiretos resultantes de doenças crônicas associadas, a má nutrição representa uma carga dupla para os sistemas de saúde, sobrecarregando esses serviços com elevados custos socioeconômicos e causando grandes impactos na população pobre e em países de baixa renda ${ }^{10}$.

Além disso, sabe-se que a atual produção de alimentos, incluindo agricultura e pecuária, é responsável por cerca de $70 \%$ de todos os gases de efeito estufa liberados no Brasil ${ }^{11}$. Esses gases estão diretamente ligados ao aquecimento global e às mudanças climáticas, que contribuem com aumento de risco para doenças crônicas e impõem desafios para a própria produção de alimentos $^{12-14}$.

$\mathrm{Na}$ raiz comum relativa aos três problemas - desnutrição, obesidade e mudanças climáticas - está a insustentabilidade dos sistemas alimentares atuais ${ }^{1,15}$. Segundo a $\mathrm{FAO}^{16}$, sistemas alimentares são elementos e atividades relacionadas à produção, processamento, distribuição, preparo, consumo e descarte de alimentos com efeitos na saúde e nas questões socioeconômicas e ambientais. Esse sistema é influenciado por diversos fatores, incluindo determinantes socioculturais, demográficos e políticos. Nesse sentido, há a necessidade de uma mudança radical nos sistemas que norteiam os modos de vida, consumo, transporte e alimentação, os quais, diferente da orientação atual, devem ser concebidos holisticamente, compreendendo o impacto na saúde dos indivíduos e as reverberações no planeta Terra. Essa mudança sustenta a urgência de uma reorientação pautada na sustentabilidade, incluindo a construção de sistemas alimentares mais resilientes, justos, saudáveis e sustentáveis; o desenvolvimento de ações sinérgicas entre os diferentes setores da sociedade; e o fortalecimento e melhoria dos sistemas de saúde, que devem integrar a nutrição como um serviço básico bem como a saúde planetária, incluindo o bem-estar e a saúde humana e do planeta ${ }^{1,17-19}$.

Considerando a emergência de ações e políticas públicas pautadas em sistemas alimentares mais saudáveis e sustentáveis, neste artigo pretende-se discutir o papel das políticas públicas de saúde, particularmente do Sistema Único de Saúde (SUS) no âmbito da Atenção Primária à Saúde (APS), no combate à sindemia global e no desenvolvimento de sistemas alimentares sustentáveis. Sem que a intenção seja esgotar o assunto, o presente artigo poderá contribuir para as discussões atuais ao refletir sobre o que vem sendo desenvolvido e o que ainda precisa ser feito. 


\section{Sistema Único de Saúde, políticas públicas e sistemas alimentares}

O Sistema Único de Saúde (SUS) foi criado em 1988 e tem o objetivo de promover acesso universal e integral à saúde para todos os brasileiros ou não, em território nacional. Ele é regido por diversos princípios organizativos e doutrinários, incluindo equidade, universalidade, integralidade, controle social e descentralização ${ }^{20}$. O SUS, longe de atuar apenas no atendimento clínico e hospitalar, é responsável também pela promoção à saúde e prevenção de doenças, vacinação, campanhas de prevenção contra doenças infecciosas, entre outras ações ${ }^{21}$. Ao longo dos últimos anos, o SUS tem realizado progressos consistentes na prestação de cuidados de saúde universais e abrangentes à população brasileira, ajudando a reduzir as desigualdades no acesso aos cuidados de saúde ${ }^{22}$.

O SUS atua junto com outras políticas, essencialmente por meio da Atenção Primária à Saúde (APS), a fim de combater a sindemia global, promovendo o controle das prevalências de obesidade e desnutrição, além de contribuir na mitigação e adaptação às mudanças climáticas ${ }^{23}$.

Nesse contexto, o governo brasileiro tem trabalhado com documentos balizadores para o planejamento, monitoramento e avaliação dos programas e políticas a fim de orientar a atuação do SUS em nível federal. Para tanto, a cada quatro anos é proposto o Plano Nacional de Saúde (PNS), que estabelece diretrizes, prioridades e metas, além de indicadores para o período. $\mathrm{O}$ PNS vigente (2020-2023) ${ }^{24}$, é apoiado por outras iniciativas de aperfeiçoamento dos controles internos e melhoria contínua no processo de governança e dentre seus objetivos prevê a ampliação e a resolutividade das ações e serviços de atenção primária de forma integrada e planejada. Visto que suas ações devem estar alinhadas às demandas da sociedade e às orientações governamentais, o plano prevê a convergência entre atores internos e externos orientadores dentre outros documentos nas Diretrizes da Conferência Nacional de Saúde, que em sua diretriz doze prevê a garantia da implementação da Política Nacional de Alimentação e Nutrição (PNAN), da Política Nacional de Segurança Alimentar e Nutricional (PNSAN) e da Política Nacional de Agroecologia e Produção Orgânica (PNAPO), incentivando a produção de alimentos ambiental, social e economicamente sustentáveis ${ }^{24}$.

A PNAN, por sua vez, reúne os esforços do Estado Brasileiro em um conjunto de políticas pú- blicas que engloba proteção, respeito e promoção aos direitos humanos à saúde e à alimentação ${ }^{25}$. Neste sentido, a PNAN se apresenta como um importante vínculo entre o setor saúde e outros relacionados ao direito humano à alimentação adequada e saudável e à segurança alimentar e nutricional (SAN) ${ }^{26}$, que fazem parte da essência do SUS para o desenvolvimento sustentável do sistema alimentar. Uma de suas metas se refere à melhoria das condições de alimentação, nutrição e saúde do povo brasileiro, por intermédio da vigilância alimentar e nutricional, da promoção de práticas alimentares adequadas e saudáveis, da prevenção e do cuidado integral dos agravos conectados à alimentação e nutriçãa ${ }^{25}$. Dessa forma, a PNAN está localizada no âmbito do SUS, contribuindo para enfrentar o atual cenário de sindemia global, além de reforçar a necessidade de ações intersetoriais que extrapolam o setor saúde.

Dialogando com a PNAN, no âmbito do Sistema Nacional de Segurança Alimentar e Nutricional (SISAN), a PNSAN prevê como uma de suas diretrizes a promoção do abastecimento e estruturação de sistemas sustentáveis e descentralizados, de base agroecológica, de produção, extração, processamento e distribuição de alimentos $^{27}$. Como se trata de uma política que alcança diferentes esferas (produção, distribuição, comercialização, acesso e consumo dos alimentos), para a sua efetiva realização é necessária a participação dos setores da saúde, agricultura, economia, educação, abastecimento, proteção, trabalho, meio ambiente, promoção social, entre outros $^{28}$.

Em consonância com tais políticas, a Política Nacional de Promoção da Saúde (PNPS) entende o desenvolvimento sustentável como referência para a formação de agendas de promoção da saúde, para adoção de estratégias e temas prioritários, sendo um tema transversal dessa política ${ }^{29}$. Para a sua efetivação, a PNPS adota como valores a solidariedade, a felicidade, a ética, o respeito à diversidade, a humanização, a corresponsabilidade, a justiça social e a inclusão social, contribuindo para o desenvolvimento de estratégias mais amplas e transformadoras que permitam que a população fique mais próxima do desenvolvimento sustentável ${ }^{29}$. A Política Nacional de Atenção Básica (PNAB), abordada aqui como APS, entende a promoção da saúde como um princípio para o cuidado em saúde para o seu processo de trabalho, destacando-se a alimentação adequada e saudável e a promoção do desenvolvimento sustentável como temas relevantes que devem ser considerados nessa abordagem ${ }^{30}$. 
Ainda no contexto de ações da gestão federal, o Ministério da Saúde publicou, em 2014, a segunda edição do Guia Alimentar para a População Brasileira ${ }^{31}$, que se constitui em uma das estratégias para implementação da diretriz de promoção da alimentação adequada e saudável que integra a PNAN. Além disso, o Guia representa um instrumento de apoio às ações de educação alimentar e nutricional do SUS, colaborando na orientação de práticas alimentares que objetivem a promoção da saúde e a prevenção de doenças associadas à nutrição, dentro de um sistema alimentar sustentável ${ }^{31,32}$, levando em consideração os impactos socioambientais do atual sistema agroalimentar vigente. Assim, o Guia Alimentar reconhece que a adoção de uma alimentação adequada, saudável e culturalmente apropriada deve atuar como promotora de sistemas alimentares socialmente e ambientalmente sustentáveis ${ }^{31}$. Neste sentido, ao orientar escolhas saudáveis e sustentáveis, esse instrumento pode ser reconhecido como uma ação de trabalho triplo, isto é, pode influenciar simultaneamente as pandemias de desnutrição e de obesidade e as mudanças climáticas ${ }^{1}$.

Tais ações foram corroboradas com o lançamento da Década de Ação pela Nutrição 2016-2025 pela Organização das Nações Unidas (ONU), na qual o Brasil foi protagonista nessas discussões, sendo o primeiro país a firmar compromissos no contexto da Década ${ }^{33}$. No mais, os compromissos assumidos também dialogam com as diretrizes da PNAN, que indicam as linhas de ação para melhoria das condições de alimentação, nutrição e saúde da população brasileira ${ }^{33}$. Nesse contexto, o Quadro 1 traz documentos publicados em âmbito nacional e internacional com recomendações para sistemas alimentares saudáveis e sustentáveis.

Conforme descrito, as políticas públicas brasileiras tentam integrar as agendas de sustentabilidade e nutrição. Contudo, a complexidade dos desafios para o alcance de objetivos essenciais, como equidade, erradicação da fome e da pobreza e combate a todas as formas de má nutrição e sustentabilidade, têm exigido uma maior integração entre as agendas de políticas públicas de saúde, agricultura e meio ambiente. Em âmbito internacional, um importante exemplo dessa articulação foi o estabelecimento dos Objetivos de Desenvolvimento Sustentável (ODS) ${ }^{40}$.

Os ODS foram criados em 2015 com o objetivo de promover o progresso e aprendizado dos oito Objetivos de Desenvolvimento do Milênio estabelecidos pela ONU, e estão alinhados ao conceito de saúde planetária, possuindo, portanto, caráter transdisciplinar ${ }^{17,40}$. Todos os ODS estão direta ou indiretamente relacionados à promoção de sistemas alimentares mais sustentáveis e à saúde da população, com destaque para os ODS 2 e 3.

O ODS 2 (Fome zero e Agricultura Sustentável) tem como meta acabar com a fome, alcançar a segurança alimentar, melhorar a alimentação e nutrição e promover a agricultura sustentável ${ }^{40}$. Para tanto, deve-se levar em consideração toda a cadeia de produção de alimentos, com o desenvolvimento de práticas agrícolas mais resilientes e de menor impacto ambiental, bem como o estabelecimento e fortalecimento de políticas de incentivo aos pequenos agricultores, incluindo mulheres, povos indígenas e agricultores familiares ${ }^{40}$. Além disso, a expansão de programas já existentes, como o PBF, a assistência técnica qualificada para pequenos produtores, a ampliação de compras públicas da agricultura familiar, o desenvolvimento de programas de educação alimentar e nutricional e medidas regulatórias para a produção e comercialização de alimentos são estratégias necessárias para que esse objetivo possa ser alcançado ${ }^{41}$.

Por sua vez, o ODS 3 (Saúde e bem-estar) prevê a garantia do acesso à saúde de qualidade e da promoção do bem-estar para toda a população. Dois pontos que devem ser destacados neste ODS são a redução da mortalidade precoce por DCNT e a diminuição do número de óbitos e de doenças decorrentes do uso de produtos químicos perigosos e da contaminação do ar, solo e água ${ }^{40}$. Esse último ponto está intimamente relacionado à insustentabilidade dos sistemas alimentares e ao uso indiscriminado de terra para a produção de monoculturas e pecuária, suscitando a necessidade de regulação e endurecimento de políticas que visem o controle do uso do solo, de fertilizantes e de agrotóxicos. No mais, cabe ressaltar que uma das estratégias deste ODS é que os sistemas de saúde sejam universais e orientados fortemente pela APS, com políticas de saúde baseadas em evidências para a gestão, mitigação e adaptação das mudanças climáticas e do meio ambiente ${ }^{40}$.

\section{Desafios do Sistema Único de Saúde}

O fortalecimento do SUS é fundamental para a promoção de sistemas alimentares mais sustentáveis. Contudo, observam-se inúmeros entraves e desafios. No âmbito da saúde, apesar da grande capilaridade do SUS e seu acesso às diversas realidades brasileiras, ainda há uma importante 
Quadro 1. Recomendações para sistemas alimentares saudáveis e sustentáveis segundo documentos publicados em âmbito nacional e internacional.

\begin{tabular}{|c|c|c|}
\hline Entidade & Documento & Recomendações \\
\hline \multicolumn{3}{|r|}{ Âmbito nacional } \\
\hline Sustentarea & $\begin{array}{l}\text { Alimentação } \\
\text { sustentável: } \\
\text { manifesto } \\
\text { Sustentarea }^{34}\end{array}$ & $\begin{array}{l}\text { Consumo preferencial de frutas, legumes, verduras, grãos integrais, } \\
\text { leguminosas e oleaginosas } \\
\text { Consumo moderado de peixe, frango, ovos e laticínios } \\
\text { Consumo reduzido de carne vermelha e processada, alimentos } \\
\text { ultraprocessados, grãos refinados e tubérculos }\end{array}$ \\
\hline $\begin{array}{l}\text { Instituto Comida } \\
\text { do Amanhã }\end{array}$ & $\begin{array}{l}\text { Diálogo Brasil: } \\
\text { Food Systems } \\
\text { Dialogues }^{35}\end{array}$ & $\begin{array}{l}\text { Acesso a alimentos saudáveis e remuneração justa para os } \\
\text { produtores de alimentos } \\
\text { Protocolos sanitários inteligentes } \\
\text { Fortalecimento da receita dos produtores de alimentos } \\
\text { Resiliência dos sistemas alimentares a futuras pandemias } \\
\text { Sistema alimentar pós-COVID alinhado à implementação dos } \\
\text { Objetivos de Desenvolvimento Sustentável } \\
\text { Aumento da informação dos consumidores sobre as origens e os } \\
\text { impactos da cadeia alimentar } \\
\text { Maior eficácia das políticas e das parcerias público-privadas para o } \\
\text { combate à fome, à insegurança alimentar e à obesidade }\end{array}$ \\
\hline $\begin{array}{l}\text { Conferência } \\
\text { Popular por } \\
\text { Direitos, Soberania } \\
\text { e Segurança } \\
\text { Alimentar e } \\
\text { Nutricional } \\
\text { (CPSSAN) }\end{array}$ & $\begin{array}{l}\text { Carta aberta } \\
\text { por sistemas } \\
\text { alimentares } \\
\text { saudáveis e } \\
\text { sustentáveis em } \\
\text { nossas cidades }^{36}\end{array}$ & $\begin{array}{l}\text { Dinamização de circuitos de abastecimento popular } \\
\text { Acesso à alimentação e enfrentamento da fome } \\
\text { Preservação dos bens comuns e da sociobiodiversidade } \\
\text { Comida como patrimônio, conhecimentos tradicionais e cultura } \\
\text { Ampliação e qualificação da atenção nutricional } \\
\text { Fortalecimento do Sistema Nacional de Segurança Alimentar e } \\
\text { Nutricional (SISAN) } \\
\text { Ordenamento urbano }\end{array}$ \\
\hline \multicolumn{3}{|r|}{ Âmbito internacional } \\
\hline $\begin{array}{l}\text { EAT-Lancet } \\
\text { Commission on } \\
\text { Food, Planet, } \\
\text { Health }\end{array}$ & $\begin{array}{l}\text { Food in the } \\
\text { Anthropocene: } \\
\text { the EAT-Lancet } \\
\text { Commission on } \\
\text { healthy diets from } \\
\text { sustainable food } \\
\text { systems }\end{array}$ & $\begin{array}{l}\text { Alimentação baseada em alimentos de origem vegetal } \\
\text { Redução do consumo de carne vermelha, grãos refinados, alimentos } \\
\text { ultraprocessados e açúcares de adição } \\
\text { Controle do uso de água potável } \\
\text { Cessação do desmatamento } \\
\text { Preservação da biodiversidade } \\
\text { Redução da poluição por nitrogênio e fósforo } \\
\text { Adoção de práticas agrícolas ambientalmente adequadas, com } \\
\text { aumento da produção de alimentos }\end{array}$ \\
\hline $\begin{array}{l}\text { Organização das } \\
\text { Nações Unidas para } \\
\text { a Alimentação e a } \\
\text { Agricultura (FAO) }\end{array}$ & $\begin{array}{l}\text { 36 }{ }^{\text {a }} \text { Conferência } \\
\text { Regional da FAO } \\
\text { para a América } \\
\text { Latina e Caribe }\end{array}$ & $\begin{array}{l}\text { Práticas comerciais baseadas em regras multilaterais } \\
\text { Políticas públicas sobre alimentação e nutrição que abordem a má } \\
\text { nutrição em todas as suas formas } \\
\text { Produção de alimentos seguros e nutritivos } \\
\text { Ampliação do investimento e uma maior cooperação entre os } \\
\text { setores público e privado para a criação de empregos e a reativação } \\
\text { econômica nos sistemas agroalimentares } \\
\text { Investigação e inovação tecnológica e digital } \\
\text { Inclusão comercial da agricultura familiar, cooperativas e pequenas e } \\
\text { médias empresas }\end{array}$ \\
\hline $\begin{array}{l}\text { Organização das } \\
\text { Nações Unidas para } \\
\text { a Alimentação e a } \\
\text { Agricultura (FAO) } \\
\text { e Instituto Nacional } \\
\text { de Pesquisa para } \\
\text { a Agricultura, } \\
\text { Alimentação e Meio } \\
\text { Ambiente (INRAE) }\end{array}$ & \begin{tabular}{|l} 
Enabling \\
sustainable \\
food systems \\
- innovators' \\
handbook \\
\end{tabular} & $\begin{array}{l}\text { Atração e engajamento de consumidores } \\
\text { Integração entre comércio, produtores e consumidores } \\
\text { Preço justo e financiamento inovador } \\
\text { Compartilhamento de saberes sobre produção sustentável de } \\
\text { alimentos } \\
\text { Advocacy }\end{array}$ \\
\hline
\end{tabular}

Fonte: Elaborado pelos autores. 
falta de integração entre as agendas de políticas públicas de saúde, agricultura e meio ambiente.

O envolvimento de todos os atores do sistema alimentar (governo, indústria e sociedade civil) é necessário para a construção de políticas públicas intersetoriais. Contudo, observa-se que no desenvolvimento de políticas há a participação de poucos agentes, o que prejudica a ampliação de visões sobre o tema. Além disso, essa participação limitada faz com que essas políticas possam atender a interesses privados em detrimento das demandas da sociedade ${ }^{42}$.

Assim, a organização horizontal dessas políticas públicas, o que permite maior integração entre elas ${ }^{43}$, é o primeiro passo para o desenvolvimento de sistemas alimentares mais sustentáveis. A participação social também é fundamental para que essas políticas possam ser efetivas, e inclui a atuação da população, organizações da sociedade civil, movimentos sociais, organizações não governamentais e entidades multilaterais ${ }^{42}$.

Além disso, o desmonte recente de políticas sociais relacionadas à distribuição de renda, redução da pobreza e promoção da SAN merece atenção. Alguns exemplos são a redução de custos de programas estruturantes, como o PBF, Programa de Aquisição de Alimentos da Agricultura Familiar e programas de incentivo à agricultura familiar ${ }^{44}$. No mais, a extinção do Conselho Nacional de Segurança Alimentar e Nutricional e o aumento do número de pessoas em situação de pobreza e vulnerabilidade social também devem ser citados como ameaças à SAN no Brasil ${ }^{45,46}$.

Dessa forma, para que seja possível garantir a SAN e a saúde da população e do planeta, são necessárias ações em níveis individuais, institucionais e políticos nacionais e internacionais (Figura 1) para que o direito da produção social da saúde, a qual transita desde a produção ao consumo de alimentos adequados e saudáveis, seja garantido a todos ${ }^{28}$. Além disso, o uso dos recursos naturais de maneira racional é essencial para que seja cumprido o artigo 225 da Constituição Brasilei$\mathrm{ra}^{47}$ : Todos têm direito ao meio ambiente ecologicamente equilibrado, bem de uso comum do povo e essencial à sadia qualidade de vida, impondo-se ao poder público e à coletividade o dever de defendê-lo e preservá-lo para as presentes e futuras gerações.

\section{Considerações finais}

O SUS tem um papel fundamental nos cuidados em relação à alimentação e nutrição, atuando na promoção e proteção da saúde, assim como na

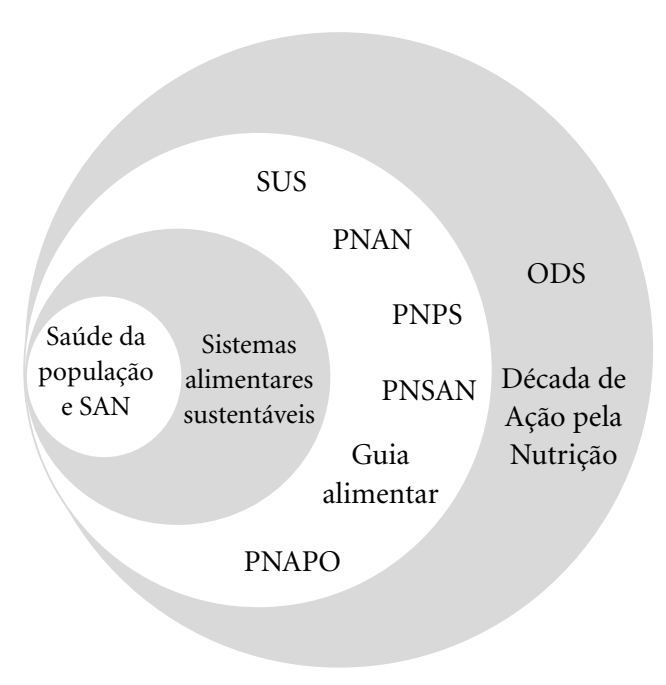

Figura 1. Relações entre Sistema Único de Saúde, sistemas alimentares e desenvolvimento sustentável.

Fonte: Elaborado pelos autores.

prevenção, diagnóstico e tratamento de doenças. As ações do SUS se mesclam ao sistema alimentar vigente, atuando como um potencial influenciador em diversas áreas do sistema como cadeia produtiva de alimentos, ambiente alimentar e consumo alimentar, com impacto na saúde, meio ambiente e economia.

Pode-se entender o SUS como uma grande ferramenta para o desenvolvimento sustentável e, para isso, há urgência que as políticas sejam convergentes e que ocorra maior integração entre as ações e interlocução entre os atores dos diversos setores, atuando de forma colaborativa. O avanço da área de saúde planetária, que estuda de maneira conjunta as ações do homem no meio ambiente e seus impactos na saúde e sustentabilidade do planeta, tem papel importante para promover a temática da sustentabilidade em políticas públicas de saúde. Além disso, a população, com acesso à educação, saúde e com agência sobre seus direitos e deveres pode exercer seu papel como cidadã, reivindicando a realização do direito humano à alimentação adequada para todos e o acesso a ambientes alimentares mais saudáveis. Assim, o SUS pode ser o articulador do desenvolvimento de um sistema alimentar mais justo, saudável e sustentável. 


\section{Colaboradores}

Todos os autores participaram da concepção do estudo, da redação do manuscrito e da revisão e aprovação da versão final a ser publicada.

\section{Referências}

1. Swinburn BA, Kraak VI, Allender S, Atkins VJ, Baker PI, Bogard JR, Brinsden H, Calvillo A, De Schutter O, Devarajan R, Ezzati M, Friel S, Goenka S, Hammond RA, Hastings G, Hawkes C, Herrero M, Hovmand PS, Howden M, Jaacks LM, Kapetanaki AB, Kasman M, Kuhnlein HV, Kumanyika SK, Larijani B, Lobstein T, Long MW, Matsudo VKR, Mills SDH, Morgan G, Morshed A, Nece PM, Pan A, Patterson DW, Sacks G, Shekar M, Simmons GL, Smit W, Tootee A, Vandevijvere S, Waterlander WE, Wolfenden L, Dietz WH. The global syndemic of obesity, undernutrition, and climate change: The Lancet Commission report. Lancet 2019; 393(10173):791-846.

2. The High Level Panel of Experts on Food Security and Nutrition (HLPE). Investing in smallholder agriculture for food security: a report by the High Level Panel of Experts on Food Security and Nutrition. Rome: FAO; 2013.

3. Duarte GR, Sampaio B, Sampaio Y. Programa Bolsa Família: impacto das transferências sobre os gastos com alimentos em famílias rurais. Rev Econ Sociol Rural 2009; 47(4):903-918.

4. Martins APB, Monteiro CA. Impact of the Bolsa Família program on food availability of low-income Brazilian families: a quasi experimental study. BMC Public Health 2016; 16(1):827.

5. Sperandio N, Rodrigues CT, Franceschini SCC, Priore SE. Impacto do Programa Bolsa Família no consumo de alimentos: estudo comparativo das regiões Sudeste e Nordeste do Brasil. Cien Saude Colet 2017; 22(6):1771-1780.

6. Instituto Brasileiro de Geografia e Estatística (IBGE) Pesquisa de orçamentos familiares 2017-2018: análise da segurança alimentar no Brasil. Rio de Janeiro: IBGE; 2020

7. Instituto Brasileiro de Geografia e Estatística (IBGE). Pesquisa nacional de saúde 2019: atenção primária à saúde e informações antropométricas. Rio de Janeiro: IBGE; 2020.

8. Coordenação-Geral de Vigilância de Agravos e Doenças Não Transmissíveis. Vigitel Brasil 2019: principais resultados. Bol Epidemiol 2020; 51(16):20-26.

9. World Health Organization (WHO). Global action plan for the prevention and control of noncommunicable diseases 2013-2020. Geneva: WHO; 2013.
10. Nilson EAF, Andrade RCS, Brito DA, Oliveira ML Custos atribuíveis à obesidade, hipertensão e diabetes no Sistema Único de Saúde, Brasil, 2018. Rev Panam Salud Publica 2020; 44:e32.

11. Azevedo TR, Costa Junior C, Brandão Junior A, Cremer MS, Piatto M, Tsai DS, Barreto P, Martins H, Sales M, Galuchi T, Rodrigues A, Morgado R, Ferreira AL, Silva FB, Viscondi GF, Santos KC, Cunha KB, Manetti A, Coluna IME, Albuquerque IR, Watanabe Junior S, Leite C, Kishinami R. SEEG initiative estimates of Brazilian greenhouse gas emissions from 1970 to 2015. Sci Data 2018; 5:180045.

12. Rossati A. Global warming and its health impact. Int $J$ Occup Environ Med 2017; 8(1):7-20.

13. Giorgini P, Di Giosia P, Petrarca M, Lattanzio F, Stamerra CA, Ferri C. Climate changes and human health: a review of the effect of environmental stressors on cardiovascular diseases across epidemiology and biological mechanisms. Curr Pharm Des 2017; 23(22):3247-3261.

14. Quam VGM, Rocklöv J, Quam MBM, Lucas RAI. Assessing greenhouse gas emissions and health co-benefits: a structured review of lifestyle-related climate change mitigation strategies. Int J Environ Res Public Health 2017; 14(5):468.

15. The High Level Panel of Experts on Food Security and Nutrition (HLPE). Nutrition and food systems. Rome: FAO; 2017.

16. Food and Agriculture Organization of the United Nations (FAO). Sustainable food systems: concept and framework. Rome: FAO; 2018.

17. Whitmee S, Haines A, Beyrer C, Boltz F, Capon AG, Dias BFS, Ezeh A, Frumkin H, Gong P, Head P, Horton R, Mace GM, Marten R, Myers SS, Nishtar S, Osofsky SA, Pattanayak SK, Pongsiri MJ, Romanelli C, Soucat A, Vega J, Yach D. Safeguarding human health in the Anthropocene epoch: report of The Rockefeller Foundation-Lancet Commission on planetary health. Lancet 2015; 386(10007): 1973-2028.

18. Haines A. Addressing challenges to human health in the Anthropocene epoch - an overview of the findings of the Rockefeller/Lancet Commission on Planetary Health. Int Health 2017; 9(5):269-271

19. Development Initiatives Poverty Research Ltd. 2020 Global Nutrition Report: action on equity to end malnutrition. Bristol: Development Initiatives; 2020. 
20. Brasil. Lei no 8.080 , de 19 de setembro de 1990. Dispõe sobre as condições para a promoção, proteção e recuperação da saúde, a organização e o funcionamento dos serviços correspondentes e dá outras providências. Diário Oficial da União 1990; 20 set.

21. Duarte E, Eble LJ, Garcia LP. 30 anos do Sistema Único de Saúde. Epidemiol Serv Saude 2018; 27(1):e00100018.

22. Castro MC, Massuda A, Almeida G, Menezes-Filho NA, Andrade MV, Noronha KVMS, Rocha R, Macinko J, Hone T, Tasca R, Giovanella L, Malik AM, Werneck H, Fachini LA, Atun R. Brazil's unified health system: the first 30 years and prospects for the future. Lancet 2019; 394(10195):345-356.

23. Ebi KL, Hess JJ, Watkiss P. Health risks and costs of climate variability and change. In: Mock CN, Nugent $\mathrm{R}$, Kobusingye O, Smith KR, organizadores. Injury prevention and environmental health. $3^{\mathrm{a}} \mathrm{ed}$. Washington: The World Bank; 2017. p. 153-169.

24. Brasil. Ministério da Saúde (MS). Plano Nacional de Saúde 2020-2023. Brasília: MS; 2020.

25. Brasil. Ministério da Saúde (MS). Política Nacional de Alimentação e Nutrição. Brasília: MS; 2012.

26. Jaime PC. Políticas públicas de alimentação e nutrição. Rio de Janeiro: Atheneu; 2019.

27. Brasil. Emenda Constitucional $n^{\circ} 64$, de 4 de fevereiro de 2010. Altera o art. $6^{\circ}$ da Constituição Federal, para introduzir a alimentação como direito social. Diário Oficial da União 2010; 4 fev.

28. Rigon SA, Schmidt ST, Bógus CM. Desafios da nutrição no Sistema Único de Saúde para construção da interface entre a saúde e a segurança alimentar e nutricional. Cad Saude Publica 2016; 32(3):e00164514.

29. Brasil. Ministério da Saúde (MS). Política Nacional de Promoção da Saúde (PNPS): Anexo I da Portaria de Consolidação $\mathrm{n}^{\circ} 2$, de 28 de setembro de 2017, que consolida as normas sobre as políticas nacionais de saúde do SUS. Brasília: MS; 2018.

30. Brasil. Ministério da Saúde (MS). Política Nacional de Atenção Básica. Brasília: MS; 2012.

31. Brasil. Ministério da Saúde (MS). Guia alimentar para a população brasileira. 2a ed. Brasília: MS; 2014.

32. Verly Junior E, Carvalho AM, Fisberg RM, Marchioni DML. Adesão ao guia alimentar para população brasileira. Rev Saude Publica 2013; 47(6):1021-1027.

33. Bortolini GA, Oliveira TFV, Silva SA, Santin RC, Medeiros OL, Spaniol AM, Pires ACL, Alves MFM, Faller LA. Ações de alimentação e nutrição na atenção primária à saúde no Brasil. Rev Panam Salud Publica 2020; 44:e39.

34. Carvalho AM, Marchioni DML. Alimentação sustentável: manifesto Sustentarea; 2019.

35. Instituto Comida do Amanhã. Diálogo Brasil: Food Systems Dialogues; 2020.

36. Conferência Nacional Popular por Direitos, Democracia, Soberania e Segurança Alimentar e Nutricional. Por sistemas alimentares saudáveis e sustentáveis em nossas cidades. Carta aberta à sociedade brasileira, aos partidos políticos, candidatos e candidatas nas eleições municipais de 2020; 2020.
37. Willett W, Rockström J, Loken B, Springmann M, Lang T, Vermeulen S, Garnett T, Tilman D, DeClerck F, Wood A, Jonell M, Clark M, Gordon LJ, Fanzo J, Hawkes C, Zurayk R, Rivera JA, De Vries W, Sibanda LM, Afshin A, Chaudhary A, Herrero M, Agustina R, Branca F, Lartey A, Fan S, Crona B, Fox E, Bignet V, Troell M, Lindahl T, Singh S, Cornell SE, Reddy KS, Narain S, Nishtar S, Murray CJL. Food in the Anthropocene: the EAT-Lancet Commission on health diets from sustainable food systems. Lancet 2019; 393(10170):447-492.

38. Organización de las Naciones Unidas para la Alimentación y la Agricultura (FAO). Transformación de los sistemas alimentarios a fin de proporcionar dietas saludables para todos. Managua: FAO; 2020.

39. Food and Agriculture Organization of the United Nations (FAO), National Research Institute for Agriculture, Food and Environment (INRAE). Enabling sustainable food systems: innovators' handbook. Rome: FAO; 2020.

40. United Nations (UN). Transforming our world: the 2030 Agenda for Sustainable Development. New York: UN; 2015.

41. Organização Pan-Americana da Saúde (OPAS). Sistemas alimentares e nutrição: a experiência brasileira para enfrentar todas as formas de má nutrição. Brasília: OPAS; 2017.

42. Marques ECL. Notas sobre redes, Estado e políticas públicas. Cad Saude Publica 2019; 35(Supl. 2): e00002318.

43. Lotta G, Favareto A. Desafios da integração nos novos arranjos institucionais de políticas públicas no Brasil. Rev Sociol Polit 2016; 24(57):49-65.

44. Ribeiro-Silva RC, Pereira M, Campello T, Aragão E, Guimarães JMM, Ferreira AJF, Barreto ML, Santos SMC. Implicações da pandemia COVID-19 para a segurança alimentar e nutricional no Brasil. Cien Saude Colet 2020; 25(9):3421-3430.

45. Santarelli M, David G, Burity V, Rocha NC. Informe Dhana 2019: autoritarismo, negação de direitos e fome. Brasília: FIAN Brasil; 2019.

46. Nery C. Agência IBGE Notícias. Extrema pobreza atinge 13,5 milhões de pessoas e chega ao maior nivel em 7 anos. [acessado 2020 out 09]. Disponível em: https://agenciadenoticias.ibge.gov.br/agencia-noticias/2012-agencia-de-noticias/noticias/25882-extrema-pobreza-atinge-13-5-milhoes-de-pessoas-e-chega-ao-maior-nivel-em-7-anos

47. Brasil. Constituição da República Federativa do Brasil. Brasília: Senado Federal; 1988.

Artigo apresentado em 20/11/2020

Aprovado em 28/05/2021

Versão final apresentada em 30/05/2021

Editores-chefes: Romeu Gomes, Antônio Augusto Moura da Silva 


\section{ERRATA}

No artigo O papel do Sistema Único de Saúde no combate à sindemia global e no desenvolvimento de sistemas alimentares sustentáveis, DOI: 10.1590/1413-812320212610.11702021

p. 4511,

onde se lia:

The role of the Brazilian Unified Health System in combating the global syndemic and in the development sustainable food systems

leia-se:

The role of the Brazilian Unified Health System in combating the global syndemic and in the development of sustainable food systems

onde se lia:

alinenutri@usp.br

leia-se:

alissondmachado@gmail.com 